\title{
Albert Fraenkel in memoriam
}

Am 22. Dezember 1938 starb im 75. Lebensjahre Albert Fraenkel, mit dessen Namen einer der größten Fortschritte in der Kreislauftherapie für immer verknüpft bleibt. Seit W. Withering^ grundlegender Veröffentlichung über die Digitalistherapie (1785) ist wohl die bedeutsamste Tat in der gesamten ärztlichen Behandlung Kreislaufkranker die Einführung der Intravenösen Strophanthintherapie durch A. Fraenkel gewesen. Die Bedeutung der intravenösen Strophanthintherapie liegt in der exakten quan-titativen Dosierung eines kristallinischen Stoffes mit Digitalis-wirkung, dessen Anwendung jedesmal mit der Genauigkeit eines Experimentes den Arzt in der Durchführung der Behandlung leitet. Dies hat Fraenkel von vorneherein erkannt und betont, und darin liegt der unschätzbare Wert dieser Behandlungsmethode. Als Fraenkel sie 1905 zuerst anwendete, war die ganze Frage der intravenösen Einverleibung von Arzneimitteln noch Neuland. Nach den ersten Versuchen von Landerer, Baccelli und Credé hat besonders Mendel in Essen seit 1902 sich für die Methode intra-venöser Arznei-Injektionen eingesetzt, deren hohen Wert Fraenkel sogleich voll zu würdigen wußte.

In Europa hat sich Fraenkels Strophanthintherapie in den verschiedensten Modifikationen seither restlos durchgesetzt, auch in ihrer von Fraenkel zuerst erkannten diagnostischen Bedeutung, in außereuropäischen Ländern noch nicht, woran zum Teil sicher eine unzweckmäßige Dosierung durch manche Aerzte

120

Albert Fraenkel in memoriam

Schuld trägt, genau so, wie dieser Umstand einst der allgemeinen Einführung der Digitalistherapie Witherings lange im Wege stand. Daß über kurz oder lang die intravenöse Strophanthin-therapie sich ihre Anerkennung in der ganzen Welt erringen muß, auch wenn sie die Digitalistherapie nie ganz verdrängen wird, scheint fraglos.

Der Mann, dem die Medizin diese geniale Leistung verdankt. wurde am 3. Juni 1864 in Nußbach in der Rheinpfalz geboren. Seine medizinische Ausbildung erhielt er in München und Straß-burg, von seinen akademischen Lehrern wurde er am meisten beeindruckt durch den Physiologen

Voíth, den Zoologen Hert-wig und die Kliniker v. Recklinghausen und Kußmaul.

Daß er selbst als junger Mensch lungenkrank war und daß er als Assistent Cornets in Berlin die aufregende Frühzeit des Tuberkulin 1890 miterlebte, mag in ihm für dieses Gebiet für Lebenszeit ein besonderes Interesse erweckt haben. Jedenfalls sind auch seine Arbeiten bezüglich der Tuberkulosefragen bedeut-sam und wegweisend.

Seit seiner Niederlassung als Arzt in Badenweiler (1893) be-gann er regelmäßig im Wintersemester im pharmakologischen Institut in Heidelberg bei von Schroder und Gottlieb zu arbeiten, und im Winter 1905/06 begann er seine Strophanthinarbeit an der Krehhchen Klinik in Straßburg durchzuführen.

Mit Beginn des Weltkrieges übersiedelte Fraenkel nach Heidelberg, wo er 1914-1919 beratender Arzt des XIV. Armeekorps und leitender Arzt des Beobachtungslazarettes Heidelberg war. Die Begründung des Tuberkulosekrankenhauses Rohrbach und des Mittelstandskrankenhauses Speyershof, sowie die Entwick-lung der Tuberkulosefürsorge in Nordbaden, war hauptsächlich 
sein Werk. Seit 1914 königlich-preußischer Professor, wurde Fraenkel ordentlicher Honorarprofessor an der Universität Heidelberg mit einem Lehrauftrag für Tuberkulose.

Dieser geniale und vorbiidliche Arzt, dieser feinsinnige Mensch wurde schließlich, wie alle jüdischen Aerzte und Wissen-schafter Deutschlands, ein Opfer der Verhältnisse. 1933 mußte er aus den von ihm begründeten beiden großen Krankenhäusern ausscheiden, 1938 wurde ihm nach 50jähriger ruhmvoller ärztlicher Tätigkeit von Staats wegen die Approbation und damit das Recht, seinen ärztlichen Beruf weiter auszuüben, entzogen.

Erst die Zukunft und die Geschichte der Medizin wird den

Leistungen Fraenkels bezüglich der Kreislauftherapie in verdienter Weise gerecht werden. Bruno Kisch, New York, 